\title{
A Model for Implementing an Interoperable Electronic Consent Form for Medical Treatment Using HL7 FHIR
}

\author{
Anna M Lackerbauer ${ }^{1,3 *}$, Alvin C Lin ${ }^{2}$, Oliver Krauss ${ }^{1}$, Jason Hearn ${ }^{3}$ and Emmanuel Helm ${ }^{1}$ \\ ${ }^{1}$ Department of Advanced Information Systems and Technology, University of Applied Sciences Upper Austria, \\ Hagenberg, Austria \\ ${ }^{2}$ Department of Surgery, Faculty of Medicine, University of Toronto, Toronto, Canada \\ ${ }^{3}$ Centre for Global eHealth Innovation, University Health Network, Toronto, Canada
}

\begin{abstract}
Background: For ethical, legal and administrative reasons, patients have to give explicit consent to a medical treatment.
\end{abstract}

Objectives: This paper identifies the design requirements for electronic treatment consent (eConsent) architecture, and subsequently proposes a model for the eConsent architecture based on the HL7 FHIR ${ }^{\circledR}$ standard.

Methods: Six requirements for the eConsent architecture were identified. A conceptual model for the system was then developed to address the identified requirements using HL7 FHIR.

Results: The proposed concept makes use of the existing

Correspondence to:

Anna Lackerbauer

University of Applied Sciences Upper Austria, Hagenberg, Austria.

E-mail: anna.lackerbauer@fh-hagenberg.at consent model of HL7 FHIR, and includes additional resources for presenting the information to the patient. Moreover, it uses the SNOMED CT terminology to enable semantic interoperability with other health information systems.

Conclusions: The proposed eConsent architecture meets the identified requirements. That said, the system is limited by the low maturity of the implemented FHIR resources and the fact that the terminology is currently inexhaustive for the use case. Custom extensions of the used FHIR resources must be considered.

\section{Keywords}

Informed consent; Health information systems; HL7; Systematized nomenclature of medicine

EJBI 2018; 14(3):37-47

Received: March 21, 2018

Accepted: May 21, 2018

Published: July 06, 2018

\section{Introduction}

This paper identifies the design requirements for electronic treatment consent (eConsent) architecture, and subsequently proposes a model for the eConsent architecture based on the HL7 FHIR standard [1]. The eConsent architecture comprises template forms, the actual treatment information, the patient consent and the signature of the patient. All four elements are represented using HL7 FHIR resources [2] and can be integrated within a FHIR ecosystem.

\subsection{Treatment Consent}

For ethical, legal and administrative reasons, patients have to give explicit consent to a medical treatment [3]. The legal requirements for a patient to be capable of giving consent to their treatment are equal across different countries: the patient must understand the steps involved in their treatment and he/she must appreciate the treatment implications $[4,5$, $6]$. Some countries additionally define a minimum age for consent $[4,5]$.

To ensure these requirements are met, the informed consent process involves multiple elements, as depicted in Figure 1. Information is first exchanged during a mandatory discussion between the patient and physician, a session in which the physician may use information materials such as printed content or videos [7]. During the discussion, the patient is educated by the physician performing the procedure about the risks, alternatives and benefits associated with the treatment [8]. Throughout the discussion, the patient is provided with the opportunity to ask questions about the upcoming procedure. Once the patient has been fully informed and their questions have been answered, he/she can decide whether he/she wants to receive the treatment. This choice must be documented and accompanied by a signature from both the patient and the treating physician. 


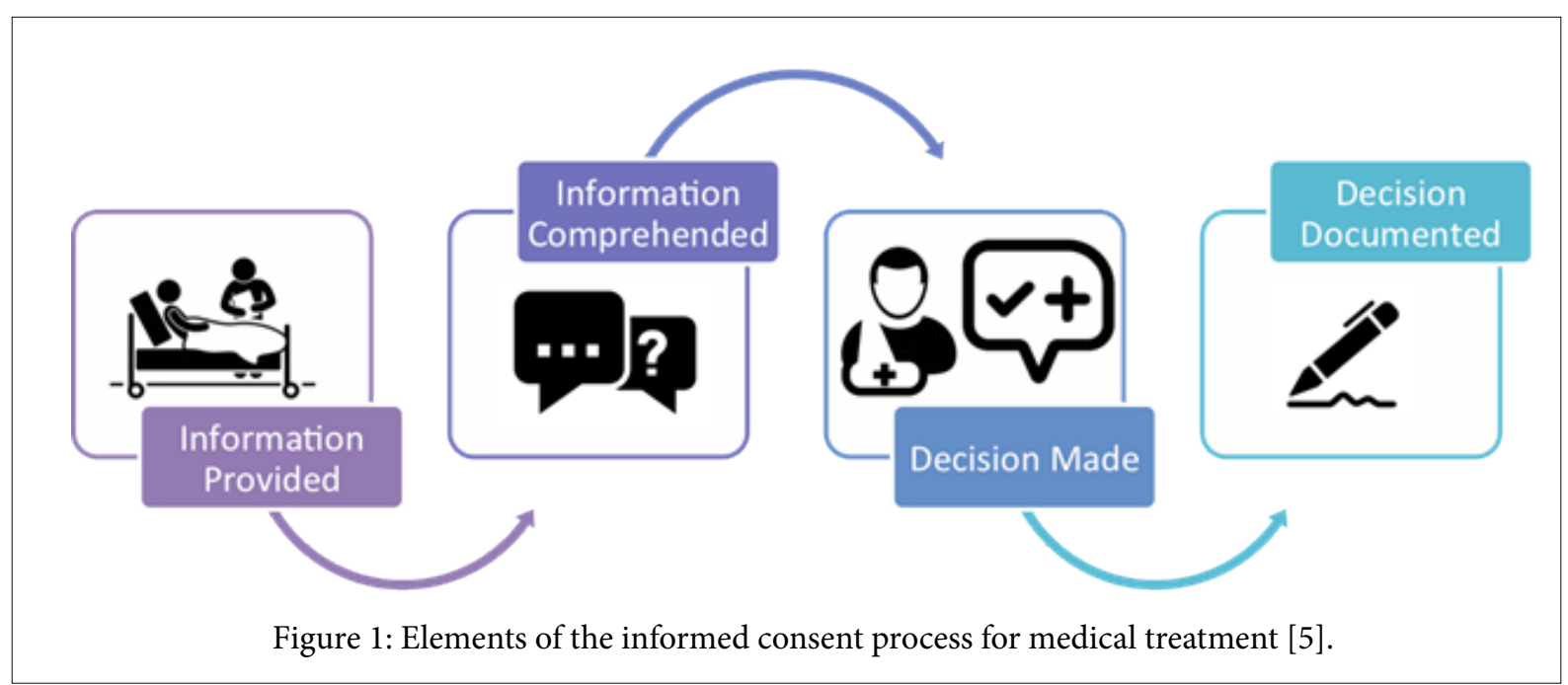

There is a large volume of work that has been done to digitize consent for research studies. As part of the data collection tool REDCap (Research Electronic Data Capture), the administration of electronic informed consent is already possible [9]. The literature proposes the usage of electronic informed consent to enhance recruitment in research studies [10].

\section{Requirements for Treatment eConsent}

Based on legal requirements, expert interviews and the academic literature, the following six requirements for the eConsent architecture were identified: simple creation, easy to understand, multi-language support, signature of various roles, rejection and withdrawal, and interoperability.

\subsection{Simple Creation}

The information that is given to the patient about the suggested treatment he/she will receive differs for each treatment. It will always contain the same, legally-required sections (i.e. risks, benefits and alternatives [8]), but the content of these sections must be defined individually for each treatment.

Within the eConsent architecture, this content should be entered into the system once for each new treatment. As many different types of treatments exist, the process of inputting new treatment information should require minimal effort. Additionally, the information input should contain all mandatory fields defined by the regulations of the government and/or institution in which the architecture is being used $[8,11]$. As an example, the mandatory section risks for the treatment information about a laparoscopic appendectomy include information about the risks that apply specifically to that procedure:

- Bleeding,

- Infection,

- Damaging neighbor structures,

- Risk of opening.

A similar requirement concerning the creation of informed consent forms for research studies was addressed by the University of California San Diego Human Research Protection Program with their implementation of an informed consent assistant [12].

\subsection{Easy to Understand}

According to Schenker et al., patients often have poor understanding of the information they receive as part of the informed consent process [8]. Hall, Prochazka and Fink, as well as Schenker et al., describe multiple ways to increase the patient's comprehension of the provided information, whereas Farrell et al. and Wilson et al. report the effect of multimedia information materials in healthcare $[3,8,13,14]$.

The treatment eConsent prototype should support the following ways to potentially increase the patient's comprehension:

- Use of simple language,

- Manageable amount of information,

- $\quad$ Use of multimedia.

\subsection{Multi-language Support}

Patients whose native language is not the primary language of the hospital can be disadvantaged due to misinterpreted information [15]. To protect patients from misinterpretation, the eConsent solution should support multilingual information provision.

\subsection{Signature of Various Roles}

If the patient does not have the capacity to provide consent himself/herself (i.e. if the legal requirements of understanding, appreciation or a possible given minimum age are not met), another person must give consent on the patient's behalf $[5,11,16]$. In Ontario, the person signing on behalf of the patient does not necessarily need to be a relative. For instance, it can also be an attorney for personal 
care or another authorized representative $[6,14]$. In the case of an emergency, treatment may be administered without consent $[5,11,17]$.

\subsection{Rejection and Withdrawal}

In the event that a patient does not want to receive a suggested treatment, they can opt to explicitly reject the treatment. It is crucial to educate the patient about the consequences of their decision and to document that this information was provided, particularly for urgent or medically-necessary treatment [5]. This is known as informed refusal. If the patient has already agreed to the treatment, he/she also has the option to withdraw their consent at any time prior to the surgical time out at the start of the surgery [18].

\subsection{Interoperability}

According to Palfrey and Gasser, interoperability and flow of information across multiple systems is a powerful tool that is crucial for success, increases innovation and fosters competition [19]. Therefore, the implementation should follow a healthcare standard and should be able to interoperate with other systems. Two use cases that demonstrate the importance of interoperability are as follows:

Patient information (i.e. the name, the date of birth and the patient's hospital record number) should be automatically obtained by an institution's electronic health record (EHR) system.

Structured, coded content describing the treatment information enables the patient and the healthcare provider to easily access and parse the details of a consent agreement.

\section{Proposed Architecture}

To ensure a standardized data model that is compatible with state of the art technologies such as REST, the chosen standard for the eConsent architecture is HL7 FHIR [1].

FHIR stands for Fast Healthcare Interoperability Resources. It is a new standard that is still under development and is currently released for trial use. The FHIR version that was used in the presented architecture is version STU3. FHIR is meant to be developer-friendly and supports widely-used standards for data-interchangeability and transfer, such as JSON, XML and HTTP. It is also an architecture that is based on the RESTful principles out of the box [1].

The resource-based standard already contains elements to model a patient's privacy consent. The treatment consent use case will be modeled by the responsible FHIR team in the future, and might change the given FHIR Consent resource accordingly [2]. This project tries to use existing resources to address the requirements of eConsent.

Figure 2 depicts the three major components required in the eConsent process:
Treatment information: This is the information that is shown to the patient about the treatment he/she will receive. This information typically includes a description about the procedure, as well as its risks, benefits and alternatives.

Consent: The Consent represents the decision a patient makes about whether or not he/she wants to receive the treatment. A later change of this decision (agreement/refusal) is also stored in this component.

Signature: To confirm the decision of the Consent, a signature of the patient or the consenting party is obtained.

Before the treatment consent process can start, the treatment-specific Treatment Information that is shown to the patient initially has to be created. As mentioned in section 2.1, there are mandatory fields that must be part of each Treatment Information. These mandatory fields are defined in the Template. The Template is modeled using the FHIR Questionnaire resource [2]. Based on one Template, there can be multiple Treatment Information instances that are modeled by the FHIR QuestionnaireResponse resource [2].

As an example, the Template could specify, that every Treatment Information of a given country or hospital (depending on the scope of the Template) must contain the elements risks, benefits and alternatives. An instance of this Template could be the Treatment Information for a laparoscopic appendectomy that specifies the risks, benefits and alternatives specifically associated with a laparoscopic appendectomy.

As part of the consent process, during the discussion between the physician and the patient, this Treatment Information will be shown to the patient as supporting material. The patient can then decide if he/she agrees or refuses the treatment based on the information he/she receives. This agreement/refusal is modeled by the FHIR Consent resource [2]. The actual signature (i.e. a picture/ scan of the signature) that is linked to the Consent resource is represented by a FHIR Provenance resource [2].

\subsection{FHIR Resource Mapping}

To address the given requirements, the FHIR resources, especially the resources representing the information that is shown to the patient, can be modeled as described in this section.

Simple creation: For each type of treatment (e.g., laparoscopic appendectomy), there should be one specific Treatment Information that includes each of the mandatory fields defined in the Template. An outline of these mandatory fields is auto-generated from the Template Questionnaire and can be displayed as the headings of a form. Similarly, the input elements are auto-generated based on the data type that 


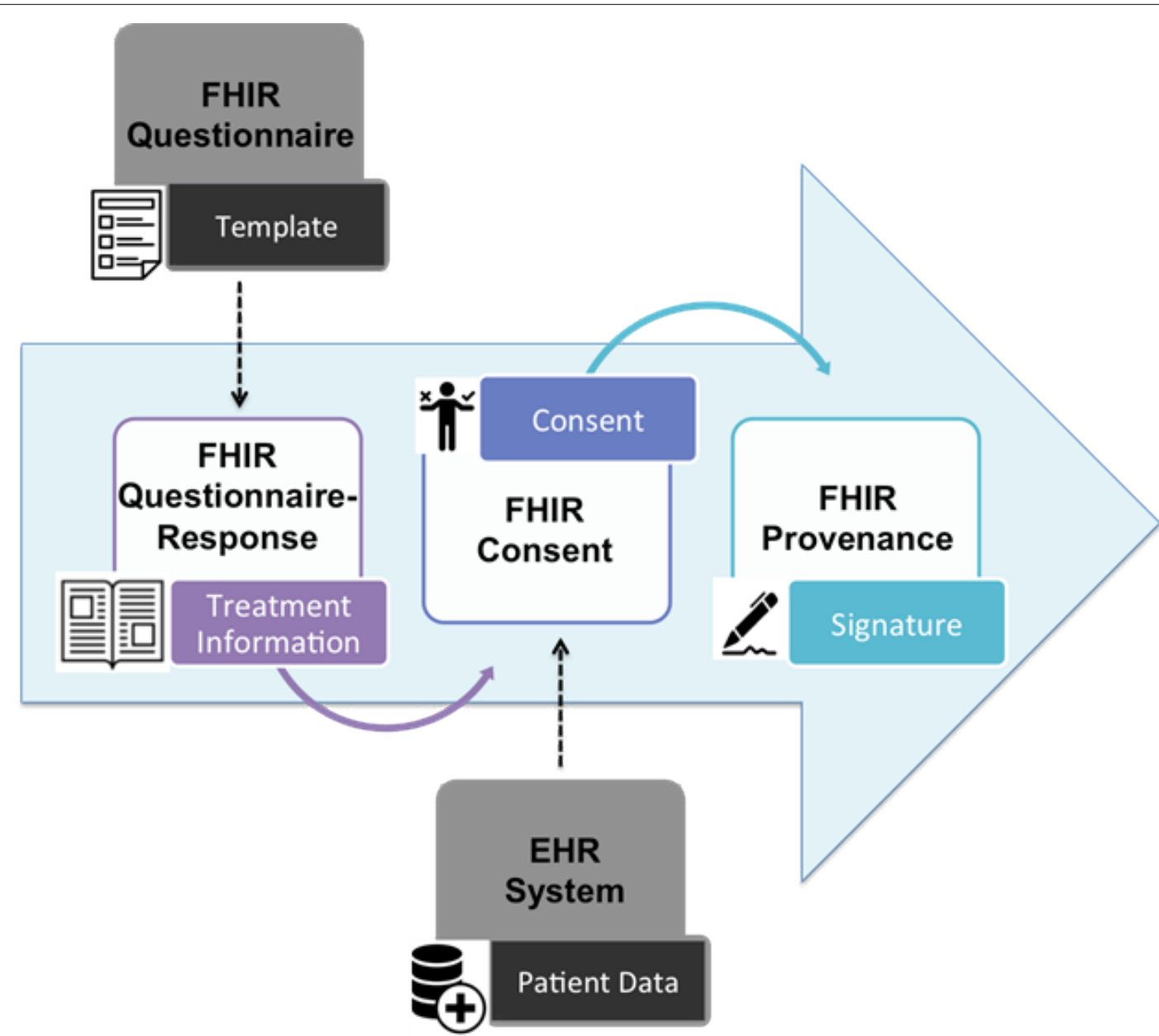

Figure 2: Proposed treatment eConsent process steps, including the data flow using existing FHIR resources.

is defined for that field in the Template Questionnaire. Depending on the required data type, the input fields can be displayed in an intuitive way, such as checkboxes or text input.

Figure 3 shows one possibility of how such a Treatment Information can be created. In this case, one can see autogenerated input fields of the data type open-choice and text [20]. The approach depicted in Figure 3 ensures that all required fields of the Template Questionnaire will be part of the resulting Treatment Information QuestionnaireResponse. As the Summary item of Potential risks is of the data type open-choice with elements predefined by a FHIR ValueSet resource [2], the user is given the option of choosing between common risks and/or creating new risks using the provided text input element.

Manageable amount of information: Too much information on the screen can overwhelm the patient. Accordingly, the content can be split into a summary and a more detailed Learn more section. As shown in Figure 4, the patient initially sees a summary of the information. On request (by selecting the Learn more button), additional information is provided.

Listing 1 shows how this item of the Questionnaire (Template) would appear as FHIR JSON format. A question of the data type group defines the overall Potential risks question with two sub- items: The Summary item for the information that is shown to the patient initially, and the Learn more item for additional information. In the auto-generated UI, this pattern can be identified by the following characteristics of the question:

- An item of type group as a parent,

- Two sub-items,

- LinkId of the first sub-item ends with "*.summary",

- LinkId of the second sub-item ends with "*.learnMore".

Use of multimedia: Similar to the previous example, the use of multimedia elements mixed with text can be handled by a question of the data type group with two sub-items, shown in Listing 2.

As the first part of the question, a textual description is required. In the second part, multiple multimedia items of type attachment can be added. The answer-item of the QuestionnaireResponse is an array, thus, on creation of the Treatment Information, the upload of multiple elements (e.g., videos and pictures) is possible. Figure 5 shows how this information, composed of text and multimedia-elements, is displayed to the patient. 


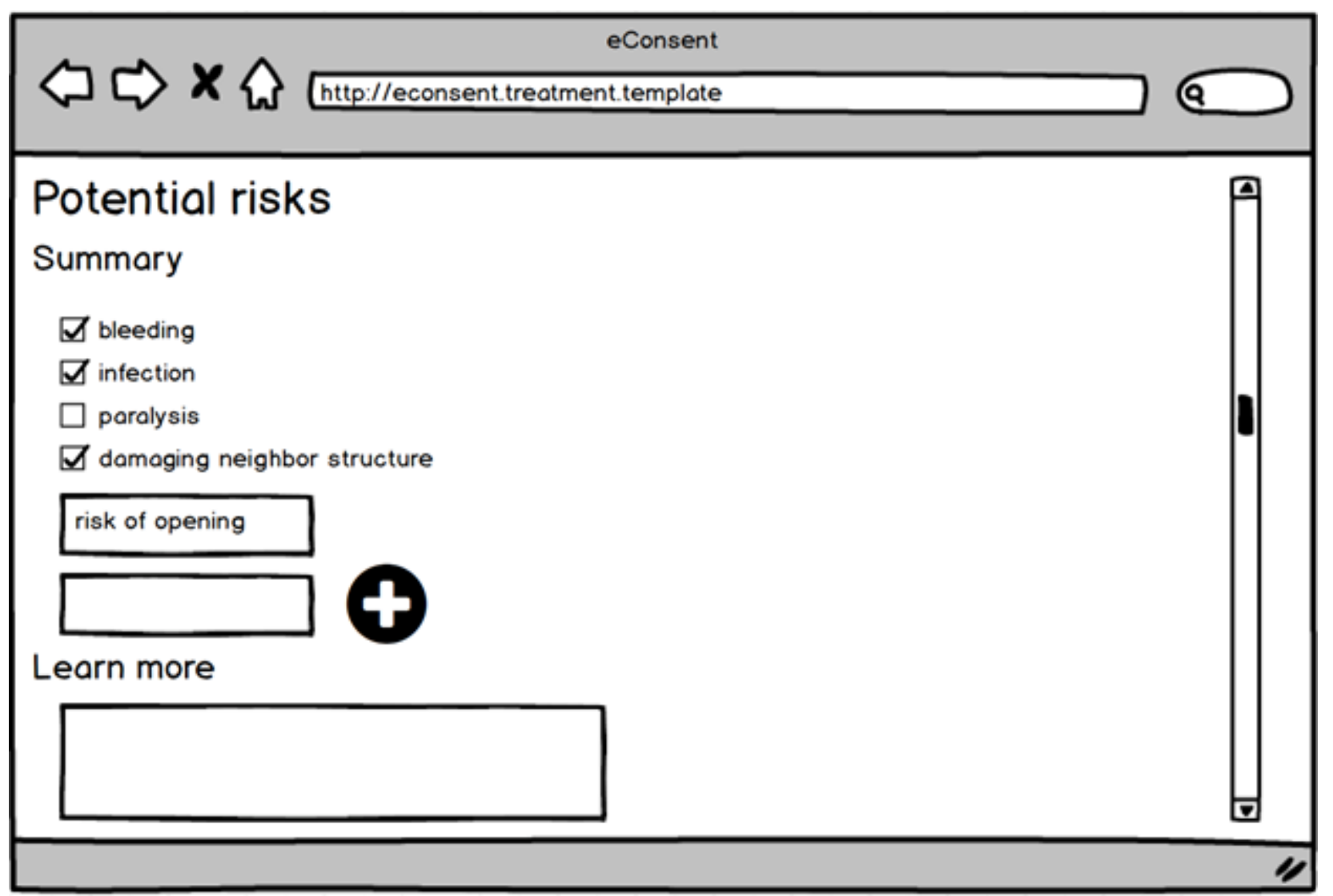

Figure 3: Example UI for the creation of Treatment Information content describing a laparoscopic appendectomy.

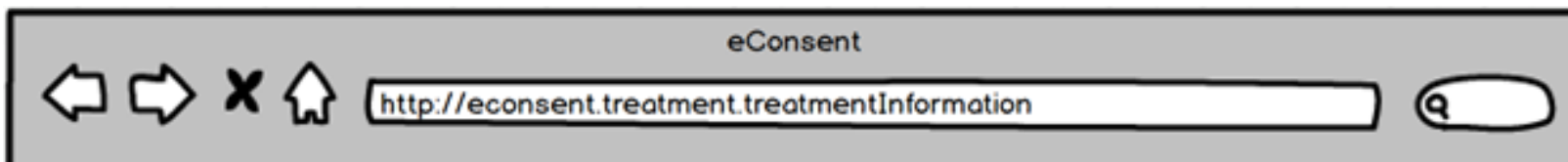

\section{Potential risks}

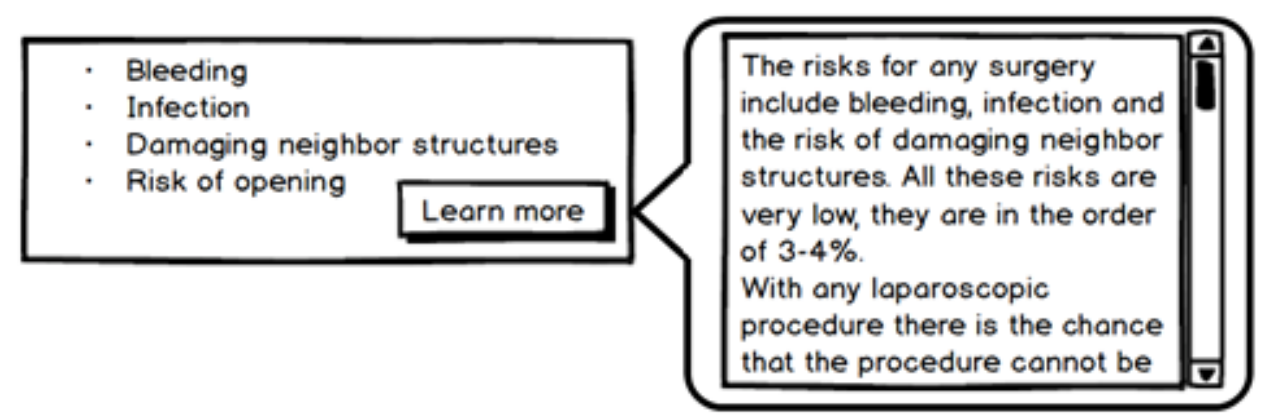

Figure 4: Example UI of a Treatment Information showing the Learn more feature. 
\{

"linkId": "econsent.treatment.risks",

"text": "Potential risks",

"type" : "group",

"item": $\quad[\{$

"linkId": "econsent.treatment.risks. summary",

"text": "Summary",

"type": "open-choice",

"options": \{

"reference" : "Valueset/risks"

\}

\},

\{

"linkId": "econsent.treatment.risks. learnMore",

"text": "Learn more",

"type" : "text"

\} ]

\}

Listing 1: Questionnaire-item representing the Learn more scenario.

\{

"linkId": "econsent.treatment.description",

"text": "Description of the procedure",

"type": "group",

"item" : $\quad[\{$

"linkId": "econsent.treatment.description.text",

"text": "Text",

"type": "text"

\},

"linkId": "econsent.treatment.description.multimedia",

"text": "Multimedia",

"type": "attachment"

\} ]

Listing 2: Questionnaire-item representing the multimedia scenario. 
Multi-language support: To achieve multi-language support, multiple resources (i.e. Questionnaire and QuestionnaireResponse) for each language can be created.

Alternatively, it may be preferable to store all Treatment Information for a given treatment, including the representations of this information in multiple languages, in a single resource. This single-resource option can be achieved by making use of the translation extension [21]. The extension can be attached to each item-element of the Questionnaire and the QuestionnaireResponse, so that both the heading and content can be translated into different languages.

Another option would be the use of an integration server, such as NextGen Connect, to deliver a requested resource in the required language. NextGen Connect, formerly known as Mirth Connect, is an open-source engine for HL7 that can be used as an integration server [22]. This option keeps the resources lightweight, similar to using multiple single-language resources, for delivery to a client application. An integration server can either manipulate the existing translation extensions, or store translations by item-numbers in the Questionnaire resources [23].

Signature of various roles: Depending on the situation, there can be a third-party individual that signs the consent form on behalf of, or in addition to, the patient. FHIR Consent and Provenance resources already support the case in which a third- party individual signs the form. This is accomplished through the element Consent.consentingParty, as well as the Provenance. agent.who reference.

Rejection and withdrawal: The Consent.status element is set to active for a consent agreement. In case of a refusal, this status is set to rejected. If the patient decides to withdraw an already active consent, the Consent resource can be updated and the status will be changed from active to rejected. Similarly, an already rejected consent can be set to active again if the patient agrees to the treatment at a later point in time [24].

Interoperability: To improve the interoperability of the proposed system, ValueSet elements can be represented by codings of a standardized terminology. Ahmadin et al. discuss the representation using narrative text of guidelines for preoperative assessment with SNOMED CT. They state, that over $70 \%$ of the used terms can be represented using that terminology [25].

Right now, SNOMED CT [26] does not offer a specific group of codings for risks as a consequence of a surgical procedure. Nevertheless, there are options to code most of these risks. Given the laparoscopic appendectomy example, the associated risks can be represented by the codes listed in Table 1 .

In some cases, such as the infection example in Table 1, more than one code can be suitable for a given risk. Other risks, such

Using a cannula (a narrow tube-like instrument), the surgeon enters the abdomen. A laparoscope (a tiny telescope connected to a video camera) is inserted through a cannula, giving the surgeon a magnified view of the patient's internal organs on a television monitor. Several other cannulas are inserted to allow the surgeon to work inside and remove the appendix. Source: www.sages.org
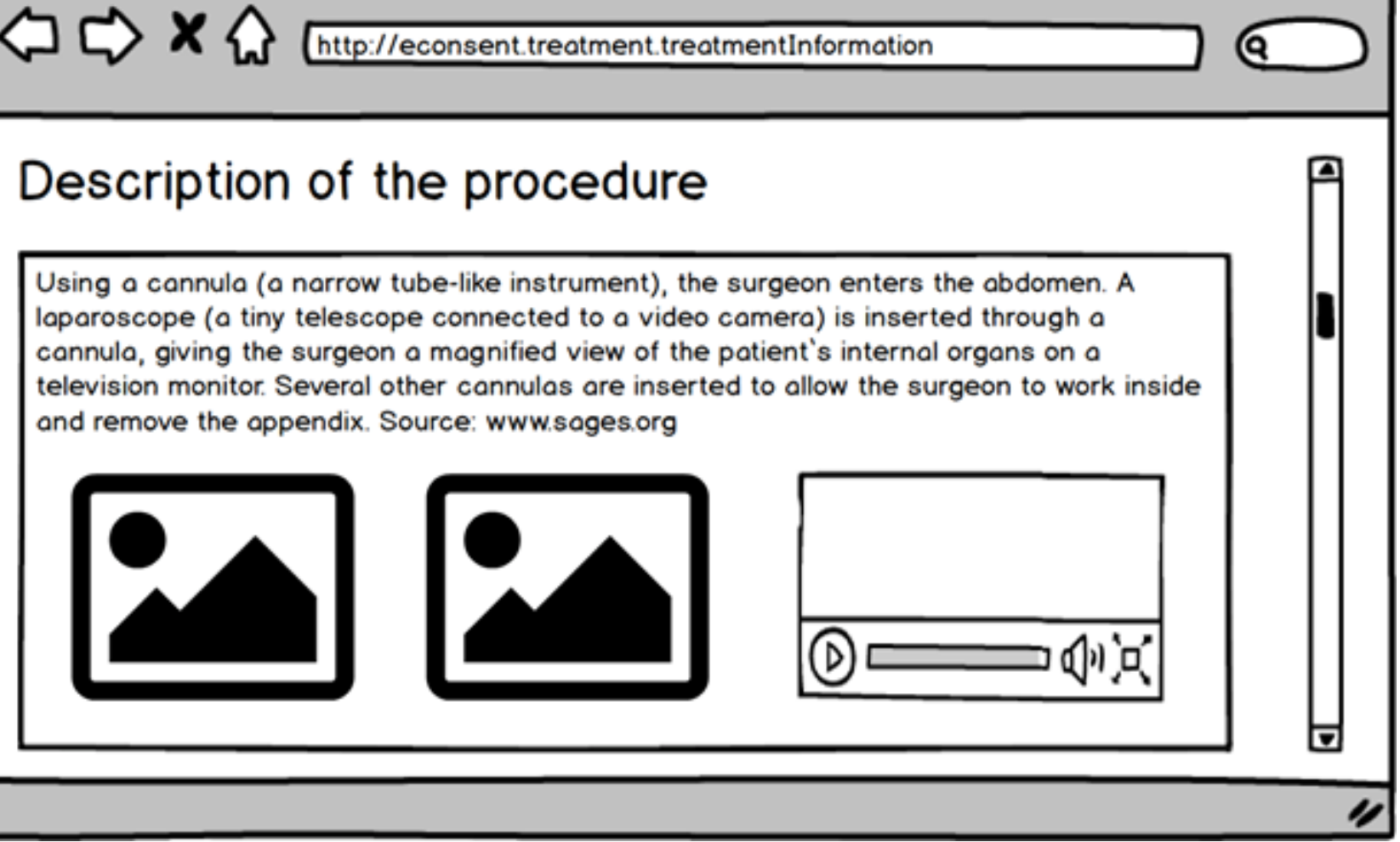

Figure 5: Example UI of Treatment Information content showing the multimedia feature. 
as the risk of opening, may not have a single representation in the terminology. For any risk without an associated terminology, the default coding shown in the last row of Table 1 can be used. However, this representation is evidently less informative given its lack of specificity.

To have a coding for every risk and to represent them in a more accurate way (i.e. including the qualifiers at risk at and perioperative), an extension of SNOMED CT might be requested (as discussed in the subsequent section).

\section{Discussion}

Some underlying conditions discussed below lead to limitations in the proposed architecture. A possible extension of the SNOMED CT terminology is also discussed. Furthermore, the use of the FHIR QuestionnaireResponse as information source is examined.

\subsection{Limitations}

To allow for compatibility of the proposed eConsent architecture with institutional EHR systems, it was assumed that institutions offer a way to integrate FHIR applications with their systems. This assumption was made on the basis that there are existing solutions that integrate FHIR with an IHE infrastructure (e.g. PIXm [27], PDQm [28], MHD [29]). That said, the number of institutions already using or planning to use an infrastructure that supports these profiles is currently unknown.

The maturity levels of the current FHIR resources must also be taken into consideration. As the FHIR standard is still under development, the maturity level describes the stability of a given resource. More specifically, a resource's maturity level is based on the types and level of review that the resource has received, and can range from 0 (draft) to 6 (normative) [30]. The implemented FHIR version, STU3, is a standard for trial use and none of the defined resources are normative before version R4 (Release 4). Accordingly, the integrated resources may change in later versions and can become incompatible with older versions. Once a resource is part of the normative standard, it is less likely to change and backward-compatibility becomes a requirement [30]. The FHIR Consent resource and the translation extension used in the proposed architecture currently possess a maturity level of 1 . Accordingly, these resources may change significantly before they become part of the normative standard.

The proposed architecture includes a step for obtaining the patient's signature electronically. This paper discusses technical considerations for the creation of a treatment eConsent architecture using HL7 FHIR. Possible legal limitations, such as cases that mandate a physical consent form or limitations based on data privacy regulations are outside the scope of this paper. Guidelines for obtaining eConsent for research studies exist and permit the use of electronic signatures if they are legally valid [31]. Unlike the informed consent process for research studies, the treatment informed consent process cannot be fully implemented electronically, as the discussion between the physician and the patient must not be replaced [7, 31]. This implies that there is no need to verify the patient's identity in a digital way, as this can be done on site by the physician.

\subsection{Terminology}

SNOMED CT does not provide a coding for all of the risks as a consequence of a given procedure. Therefore, additional concepts will have to be requested. A potential existing parent element is shown in Table 2.

It might be misleading to classify risks under the parent medical accidents. An alternative is requesting additional concepts under a new subset (e.g., suggesting the subset concept name at risk for perioperative complications) of the parent finding of at risk, as shown in Table 3.

\subsection{FHIR QuestionnaireResponse as Information}

\section{Source}

The proposed architecture makes uses of the FHIR QuestionnaireResponse resource to represent the content of the Treatment Information that is shown to the patient. Proposals concerning the hierarchy and design of the Questionnaire items (i.e. the Learn more and the multimedia scenarios that are depicted in Section 3.1) can be used to generate the UI in a more user-friendly way.

Table 1: Possible SNOMED CT representation for treatment risks.

\begin{tabular}{lll}
\hline Risk (Narrative) & SNOMED CT Coding & Description \\
\hline Bleeding & 242996005 & Accidental hemorrhage during medical care (finding) \\
Infection & 12246311000119109 & Infection following procedure (disorder) \\
Infection & 762611002 & Infection of organ surgical site following surgical procedure (disorder) \\
Infection & 413590008 & At risk of healthcare associated infection (finding) \\
$\begin{array}{l}\text { Damaging neighbor } \\
\text { structures }\end{array}$ & 409031004 & At risk for perioperative injury (finding) \\
$\begin{array}{l}\text { Risk of opening } \\
\text { General (Default) }\end{array}$ & - & - \\
\hline
\end{tabular}


Table 2: Possible SNOMED CT parent for treatment risks: Medical accidents.

\begin{tabular}{ll}
\hline SNOMED Code & Definition \\
\hline 269691005 & Medical accidents to patients during surgical and medical care (event) \\
\hline
\end{tabular}

Table 3: Possible SNOMED CT parent for treatment risks: Finding of at risk.

\begin{tabular}{ll} 
SNOMED Code & Definition \\
\hline 281694009 & Finding of at risk (finding) \\
\hline
\end{tabular}

The aim of theFHIRQuestionnaireResponse resource is to capture a set of answers given to a specific FHIR Questionnaire resource [2]. The proposed usage of the FHIR QuestionnaireResponse resource, to represent compiled information that follows a defined outline and that can be displayed in an auto-generated UI, is not intended by the standard. With the proposed usage of questionnaire items to split up long content and to combine multimedia elements with text, however, this representation is possible. That being said, there are some notable restrictions with the proposed approach:

1. Multimedia: Text and multimedia elements can be mixed within a single section (see multimedia scenario in Section 3.1.2), however, their order is strictly defined by the item order of the FHIR Questionnaire. This reduces the flexibility with which the text and multimedia elements can be combined. As an example, the order of the items depicted in Listing 2 requires a text input first, followed by at least one multimedia element. Accordingly, the generated UI must show the text prior to any multimedia elements. Allowing for multiple alternating text and multimedia items in the Questionnaire would be an enhancement, but would still fail to provide full control over the order of the content elements. Accepting answer elements of several data types (i.e. by changing the cardinality of the Questionnaire.item.type element from $1: 1$ to $1:^{*}$ ) is one possible solution.

2. Text emphasis: The proposed architecture allows for the input of plain text, but it does not allow the user to emphasize content (e.g. bold or italic words). Taking a markup language as the input for an answer of the data type text can remedy this limitation. Another option is the expansion of the ValueSet itemtype [20] to a markup type.

The primary intention of the FHIR QuestionnaireResponse resource differs from the use case proposed in this paper. The introduction of custom extensions or the use of other information sources must be taken into consideration to address the needs of emphasized and flexible content.

\section{Conclusion and Future Work}

The existing consent model of HL7 FHIR that currently focuses on privacy consent provides the basic elements that are needed to model the electronic treatment consent use case. The FHIR Questionnaire and QuestionnaireResponse resources allow the representation of different structures. These two resources contain the elements necessary to auto-generate a UI containing a form with given headings and dynamic input fields. A notable limitation of this approach is that the order of elements is strict and not flexible when it comes to mixing data types such as text and images within a single section. Furthermore, the proposed system does not support text emphasis, which is needed to further increase content readability.

The usage of codings for some common concepts (e.g., risks) can increase the interoperability of the application. Missing concepts will be requested to be part of the SNOMED CT terminology.

A web-based, open-source prototype that auto-generates the UI for clinicians (to enter new Treatment Information) and patients (to sign the consent) are currently under development. The open-source project is available at https://github.com/ehealthinnovation/eConsent. When this prototype is finished, other types of consent, such as informed consent for research studies, will be considered as a continuation of the open-source project. Expert feedback will also be obtained, including feedback from surgeons as the main users of the treatment consent, as well as feedback from researchers concerning the planned research study consent implementation.

Lastly, experiences will be shared with the HL7 FHIR Consent Directive Project working group. The working group will also be approached for feedback regarding usage of the draft standard.

\section{Acknowledgement}

The work is motivated by Dr. Lin's intention of making the consenting process easier for patients, and was realized in cooperation with the University of Applied Sciences Upper Austria. The Centre for Global eHealth Innovation kindly supported the project by providing resources for the development of an open-source prototype. Many thanks to William Chou as the front-end developer of the open-source project, as well as to James Agnew as the founder of the opensource project HAPI FHIR that is used as a basis for the backend implementation. Also, thanks to Linda Parisien as part of the SNOMED CT International Member Forum for giving advice on the usage and possible extension of SNOMED CT. 
Glossary

\begin{tabular}{ll}
\hline EHR & Electronic Health Record. \\
FHIR & $\begin{array}{l}\text { Fast Healthcare Interoperability Resources. } \\
\text { Standards framework created by HL7. }\end{array}$ \\
HL7 & $\begin{array}{l}\text { Health Level 7. Organization that develops } \\
\text { standards concerning electronic health } \\
\text { information. }\end{array}$ \\
HTTP & $\begin{array}{l}\text { Hypertext Transfer Protocol. A protocol that is } \\
\text { used for communication in the web. }\end{array}$ \\
& $\begin{array}{l}\text { Integrating the Healthcare Enterprise. An } \\
\text { initiative to improve information sharing in } \\
\text { healthcare by promoting the coordinated use of } \\
\text { standards. }\end{array}$
\end{tabular}

JSON JavaScript Object Notation. A lightweight format for data exchange.

REST Representational State Transfer. A style of architecture to develop web services.

\section{RESTful A REST-compliant system. \\ SNOMED Systematized Nomenclature of Medicine - \\ CT Clinical Terms. A healthcare terminology system including terms, synonyms and codes.}

STU3 Standard for Trial Use 3. Current official FHIR version that was published on April 19, 2017.

UI User Interface.

XML Extensible Markup Language. A markup language that can be used to store and exchange data.

\section{References}

[1] HL7 International. FHIR. FHIR Release 3 (STU). 2017. https://www.hl7.org/fhir

[2] HL7 International. Resource Index. FHIR Release 3 (STU). https://www.hl7.org/fhir/resourcelist.html

[3] Hall D, Prochazka A, Fink A. Informed Consent for Clinical Treatment. Can Med Assoc J. 2012; 184: 533-540.

[4] European Union Agency for Fundamental Rights. Consenting to Medical Treatment without Parental Consent. 2017. http:// fra.europa.eu/en/publication/2017/mapping-minimum-agerequirements/consent-medical-treatments

[5] Canadian Medical Protective Association. Consent - A Guide for Canadian Physicians. 2016. https://www.cmpa-acpm.ca/ en/advice-publications/handbooks/consent-a-guide-forcanadian-physicians

[6] College of Physicians and Surgeons of Ontario. Determining Capacity to Consent. 2007. https://www.cpso.on.ca/uploadedFiles/policies/policies/policyitems/capacity_consent_july07dialogue.pdf
[7] Canadian Medical Protective Association. Documenting Consent. 2018. https://www.cmpa-acpm.ca/serve/docs/ ela/goodpracticesguide/pages/communication/Informed_Consent/documenting-e.html

[8] Schenker Y, Fernandez A, Sudore R, Schillinger D. Interventions to Improve Patient Comprehension in Informed Consent for Medical and Surgical Procedures. Med Decis Making. 2010; 31: 151-173.

[9] Vanderbilt University Medical Center. REDCap About. 2018. https://projectredcap.org/about

[10]Sanderson I, Obeid J, Madathil K, Gerken K, Fryar K, Rugg D, et al. Managing Clinical Research Permissions Electronically: A Novel Approach to Enhancing Recruitment and Managing Consents. Clinical Trials. 2013; 10: 604-611.

[11] National Health Service. Consent to Treatment. 2016. https://www.nhs.uk/conditions/consent-to-treatment

[12] UCSD Human Research Protection Program. Informed Consent Assistant. 2018. https://irb.ucsd.edu/consent. shtml

[13] Farrell E, Whistance R, Phillips K, Morgan B, Savage K, Lewis V, et al. Systematic Review and Meta-Analysis of Audio-Visual Information Aids for Informed Consent for Invasive Healthcare Procedures in Clinical Practice. Patient Educ Couns. 2014; 94: 20-32.

[14] Wilson E, Makoul G, Bojarski E, Bailey S, Waite K, Rapp $\mathrm{D}$, et al. Comparative Analysis of Print and Multimedia Health Materials: A Review of the Literature. Patient Educ Couns. 2012; 89: 7-14.

[15] Schenker Y, Wang F, Selig S, Ng R, Fernandez A. The Impact of Language Barriers on Documentation of Informed Consent at a Hospital with On-Site Interpreter Services. J Gen Intern Med. 2007; 22: 294-299.

[16] University of Innsbruck. Treatment Contract - Medical Liability. 2018. https://www.uibk.ac.at/zivilrecht/buch/ kap10_0.xml?section-view=true;section=2

[17] Government of Ontario. Health Care Consent Act. 1996. https://www.ontario.ca/laws/statute/96h02\#BK27

[18] World Health Organization. Surgical Safety Checklist. 2008. http://www.who.int/patientsafety/safesurgery/ tools_resources/SSSL_Checklist_finalJun08.pdf?ua =1

[19] Palfrey J, Gasser U. Interop: The Promise and Perils of Highly Interconnected Systems. New York: Basic Books; 2012. p. 8-111.

[20]HL7 International. ValueSet Item-Type. FHIR Release 3 (STU). 2017. https://www.hl7.org/fhir/valueset-itemtype.html 
[21]HL7 International. Extension: Translation. FHIR Release 3 (STU). 2017. http://hl7.org/fhir/extension-translation.html

[22] NextGen Healthcare. NextGen ${ }^{\oplus}$ Connect Integration Engine. 2018. https://www.nextgen.com/products-and-services/integration-engine

[23] Cerlinca M, Turcu C, Cerlinca T, Prodan R. HL7 Messaging Engine with Customizable Translation System. Adv Electr Comp Eng. 2010; 10: 98-101.

[24]HL7 International. Consent State Codes. FHIR Release 3 (STU). 2017. http://hl7.org/fhir/valueset-consent-state-codes.html

[25] Ahmadian L, Cornet R, de Keizer N. Facilitating PreOperative Assessment Guidelines Representation Using SNOMED CT. J Biomed Inform. 2010; 43: 883-890.

[26] SNOMED International. The Global Language of Healthcare. SNOEMD CT. 2018. https://www.snomed.org/snomed-ct

[27]IHE. Patient Identifier Cross-Reference for Mobile (PIXm). Integrating the Healthcare Enterprise IHE IT Infrastructure
Technical Framework Supplement. 2017. http://ihe.net/ uploadedFiles/Documents/ITI/IHE_ITI_Suppl_PIXm.pdf

[28]IHE. Patient Demographics Query for Mobile (PDQm). Integrating the Healthcare Enterprise IHE IT Infrastructure Technical Framework Supplement. 2017. http://ihe.net/ uploadedFiles/Documents/ITI/IHE_ITI_Suppl_PDQm.pdf

[29]IHE. Mobile Access to Health Documents (MHD). Integrating the Healthcare Enterprise IHE IT Infrastructure Technical Framework Supplement. 2017. https://www.ihe. net/uploadedFiles/Documents/ITI/IHE_ITI_Suppl_MHD. pdf

[30]HL7 International. Version Management Policy. FHIR Release 3 (STU). 2017. https://www.hl7.org/fhir/versions. html

[31]FDA. Use of Electronic Informed Consent Questions and Answers. Guidance for Institutional Review Boards, Investigators, and Sponsors. 2016. https://www.fda.gov/ downloads/drugs/guidances/ucm436811.pdf 